\title{
Influence of Saturation Phenomena on Laser-Excited Atomic Fluorescence Flame Spectrometry
}

\author{
J. Kuhl, S. Neumann, and M. Kriese \\ Carl Zeiss, Forschungsgruppe, Oberkochen \\ (Z. Naturforsch. 28 a, 273-279 [1973]; received 29 December 1972)
}

\begin{abstract}
Using a simple rate equation model, the laser power density $I_{\mathrm{c}}$ necessary to reach $50 \%$ of the saturation limited population of the excited atomic level under typical flame conditions is calculated. For $\mathrm{Na}$ atoms aspirated into the flame a saturating power density for irradiation with a narrow dye laser line (bandwidth $0.033 \AA$ ) of $I_{\mathrm{c}} \sim 0.4 \mathrm{~kW} / \mathrm{cm}^{2}$ was determined. With the aid of a dye laser with an appropriate laser power density, analytical curves for $\mathrm{Na}$ were measured yielding a detection limit of $0.2 \mathrm{ng} / \mathrm{ml}$. This sensitivity is comparable with the best results obtained by atomic emission flame spectrometry.
\end{abstract}

\section{Introduction}

Use of atomic fluorescence (AF) spectrometry as an analytical tool for trace element analysis has been described in several comprehensive review articles 1,2 . Recently, the application of pulsed tunable dye lasers for fluorescence excitation in flames and vapors was studied by a number of different groups $3,4,5$. Compared with conventional light sources dye lasers offer the advantage of high spectral power density, narrow bandwidth, small angle of emission, tunability over broad spectral ranges and a small duty cycle, i.e. a small on-to-off time of the source. On account of these qualities long linear analytical curves, low limits of detection in AF, improvement in signal-to-noise ratios and reduction of flame background noise are obtainable as the first initial experiments have shown.

As the spectral power density of dye lasers is sufficiently high to get saturation of electronic transitions in atoms, the laser induced fluorescence signal will be limited to values which are independent of the irradiance level and are determined by parameters of the irradiated atomic species alone. Therefore, the analytical sensitivity of the fluorescence method may be strongly diminished by the influence of unspecific scattering of laser photons within the flame, if the population of the excited level approaches the saturation. We have tried to estimate the laser output power necessary to achieve saturation conditions in resonance fluorescence by a simple rate equation model and compared these results with experimental values, measured for the Na- $\mathrm{D}_{2}$ line.

\section{Theoretical considerations}

The intensity absorbed by an atomic vapor is generally calculated from the Lambert-Beer law:

$$
I_{\mathrm{abs}}=I_{0}(1-\exp (-\alpha \sigma N L)),
$$

$I_{0}=$ incident irradiance $\left(\mathrm{W} / \mathrm{cm}^{2}\right)$, $\alpha=$ parameter which accounts for the reduction of the absorption cross section due to polarization of the exciting radiation,

$\sigma=$ absorption cross section $\left(\mathrm{cm}^{2}\right)$,

$N=$ number of absorbing atoms $/ \mathrm{cm}^{3}$,

$L=$ thickness of absorbing layer $(\mathrm{cm})$.

If a high intensity laser beam is used for irradiation, the dependence of the optical absorption coefficient on the incident light flux has to be taken into account. Due to the high spectral radiance of the pumping source, a reasonable population of the excited atomic energy level is built up in competition with different relaxation processes, i.e. radiative (spontaneous and stimulated) and non-radiative (collisional quenching) transitions. The irradiance level at which saturation becomes appreciable and the residual absorption of the partially bleached ground state may be estimated by a formalism described by HERCHER ${ }^{6}$ or GIRES ${ }^{7}$ for the analysis of saturable filters.

The irradiated atom can be characterized by a simple 3 energy level scheme (Figure 1). Levels 1 and 2 are coupled by the irradiation. A third intermediate level may describe another state of the atom, which is connected to the first two levels via radiative and nonradiative transitions. Taking into account the induced emission of photons from the excited level, the number

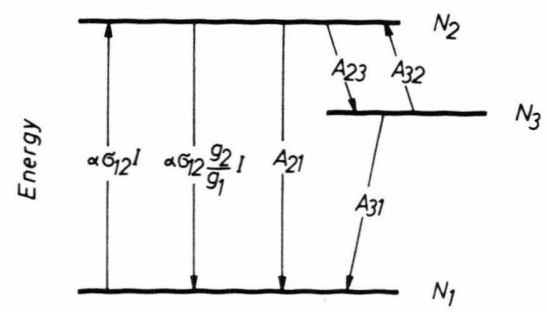

Fig. 1. Three-level energy scheme showing the excitation and decay rates which were considered for the calculation of the saturation irradiance. 
of photons absorbed in a small layer of thickness $\mathrm{d} x$ is given by:

$$
\mathrm{d} I=-\alpha \sigma_{12}\left[N_{1}-\left(g_{2} / g_{1}\right) N_{2}\right] I \mathrm{~d} x,
$$

$\sigma_{12}$ cross section for absorption,

$g_{1}, g_{2}$ statistical weights of levels 1 and 2 ,

$N_{i}$ population density of level $i, \sum_{i=1}^{3} N_{i}=N=$ total number of absorbing atoms $/ \mathrm{cm}^{3}$.

The temporal behavior of the system during illumination may be described by a set of rate equations:

$$
\begin{aligned}
\left(\mathrm{d} N_{1} / \mathrm{d} t\right)= & -\alpha \sigma_{12} I\left[N_{1}-\left(g_{2} / g_{1}\right) N_{2}\right]+N_{2} A_{21}+ \\
& +N_{3} A_{31} \\
\left(\mathrm{~d} N_{2} / \mathrm{d} t\right)= & \alpha \sigma_{12} I\left(N_{1}-\left(g_{2} / g_{1}\right) N_{2}\right)-A_{23} N_{2} \\
& +A_{32} N_{3}-A_{21} N_{2}
\end{aligned}
$$

$\left(\mathrm{d} N_{3} / \mathrm{d} t\right)=-A_{31} N_{3}+A_{23} N_{2}-A_{32} N_{3}$.

$A_{i j}=a_{i j}+z_{i j}=$ transition probabilities for spontaneous emission $\left(a_{i j}\right)$ and collision induced transitions $\left(z_{i j}\right)$.

If the time of irradiation is not too short compared with the relaxation times of the irradiated system, a steady state is reached. Assuming that the three levels shown are the only ones populated, the steady state population of level 2 is defined by

$$
\begin{aligned}
N_{2} & =\frac{N I}{\left[1+\left(g_{2} / g_{1}\right)+\left(A_{23} /\left(A_{31}+A_{32}\right)\right)\right] I+\left(A / \alpha \sigma_{12}\right)} \\
& =(N / B)\left(I /\left(I+I_{\mathrm{c}}\right)\right)
\end{aligned}
$$

where $B=1+\left(g_{2} / g_{1}\right)+\left(A_{23} /\left(A_{31}+A_{32}\right)\right)$ and

$I_{\mathrm{c}}=\left(A / \alpha \sigma_{12} B\right) ; \quad A=A_{21}+A_{31}\left(A_{23} /\left(A_{31}+A_{32}\right)\right)$.

The parameter $I_{\mathrm{c}}$ defines a critical photon irradiance for which the population of the excited level 2 reaches $50 \%$ of its limiting value $N / B$. This irradiance, which we shall call saturation irradiance, decreases with increasing optical absorption cross section and increases with decreasing lifetimes of the excited atomic levels.

The intensity $I_{\mathrm{T}}$ transmitted by the irradiated vapor sample can be calculated from

$$
\ln \left(I_{\mathrm{T} /} / I_{0}\right)=-\sigma_{12} N L-\left(\left(I_{\mathrm{T}}-I_{0}\right) / I_{\mathrm{c}}\right),
$$

$L=$ thickness of absorbing layer.

For $I_{0} \ll I_{\mathrm{c}}$ (IV) passes into the well known Lambert-Beer law.

As $I_{\mathrm{T}} / I_{0}=T$ is the transmission of the irradiated sample and $\ln T_{0}=-\sigma_{12} N L$ defines the small signal transmission (IV) is equivalent to

$$
\ln \left(T / T_{0}\right)=(1-T)\left(I_{0} / I_{\mathrm{c}}\right) .
$$

Figure $2 \mathrm{a}$ shows the population of the excited level 2 as a function of the irradiance $I_{0}$ for different values of the parameters $I_{\mathrm{c}}$ and $B$, whereas increase in transmission with increasing irradiance is illustrated in Fig. $2 \mathrm{~b}$ for different values of small signal absorption $T_{0}$. Some rules may be derived from these considerations for the selection of laser output power for laser excited flame fluorescence spectrometry.

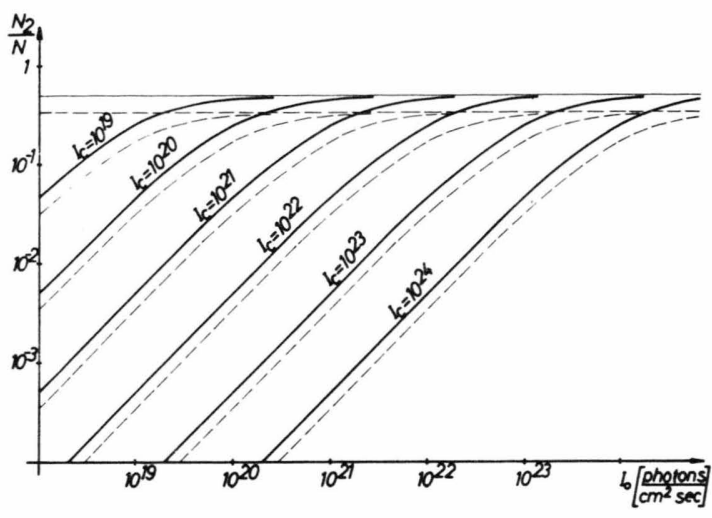

Fig. 2a. Fraction of excited atoms of a vapor sample irradiated with $I_{0}$ photons $/ \mathrm{cm}^{2} \cdot \mathrm{sec}$ for different saturation irradiances $I_{\mathrm{c}}$ (photons $/ \mathrm{cm}^{2} \cdot \mathrm{sec}$ ) of the vapor and parameters $B$ : solid curves $B=2$, dashed curves $B=3$. All curves with $B>2$ are shifted to lower values of $\log \left(N_{2} / N\right)$ about $\log (B / 2)$. (Definitions of the parameters $I_{c}$ and $B$ see text).

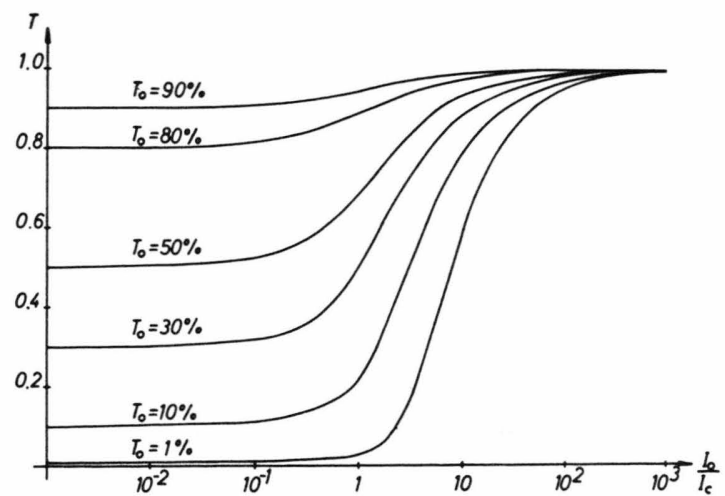

Fig. 2b. Theoretical transmission curves for a three-level saturable atomic vapor sample illuminated by intense laser radiation of varying power density. $T_{0}=$ transmission for small irradiance levels $I_{0} \ll I_{\mathrm{c}}$.

I. A limiting value of the excited atom fraction (and of the fluorescence signal) is asymptotically approached as the irradiance level overcomes the saturation irradiance $I_{\mathrm{c}}$. As a further linear increase of the noise signal due to scatter of laser photons from aerosols, molecules, and refractive index variations within the 
flame for $I_{0}>I_{\mathrm{c}}$ have to be expected, an irradiance level between $0.5 I_{\mathrm{c}}$ and $10 I_{\mathrm{c}}$ should yield an optimum of signal to noise ratio and minimum detection limits.

II. For analyte concentrations for which the fluorescence signal is large compared with the unspecific scattering signal, the influence of laser output power fluctuations on the fluorescence signal may be strongly reduced by using an irradiance $I>I_{\mathrm{c}}$ for excitation. The effect of reabsorption on the fluorescence signal is also less severe because the transmission of the sample is increased by bleaching of the ground state.

III. The fluorescence signal is the spontaneous emission in all directions equal to $N_{2} a_{21}$ photons $/ \mathrm{cm}^{3} \mathrm{sec}$. Stimulated emission occurs only in the direction of the laser beam. If the irradiation time $\Delta t$ is long compared with the relaxation times of the irradiated atoms, a steady state is reached. The total number of fluorescence photons is then proportional to $N_{2} \Delta t / \tau^{\mathrm{sp} *}$. As the population density $N_{2}$ is limited by the saturation effect, further increase in the number of observable fluorescence photons is only possible by stretching the laser pulse length. Therefore, flashlamp-pumped dye lasers with pulse lengths of the order of $10^{-6} \mathrm{sec}$ seem to be superior to $N_{2}$-laser pumped systems which have typical pulse lenghts of less than $5 \cdot 10^{-9} \mathrm{sec}$.

IV. Deviations of absorption and transmission of a vapor sample from the values calculated by the Lambert-Beer law are already observed for $I \approx 0.01 I_{\mathrm{c}}$. If for $I>0.01 I_{\mathrm{c}}$ the evaluation of the data neglects the bleaching of the ground state, the calculated concentration is too small. For $I \gg I_{c}$ the transmission measurement of a sample is no longer a suitable analytical method to determine concentrations.

\section{Experimental}

The experimental setup is shown schematically in Figure 3. Details of the flashlamp-pumped dye laser have been described comprehensively 8,9,10. Therefore, only some characteristic data are summarized in Table 1.

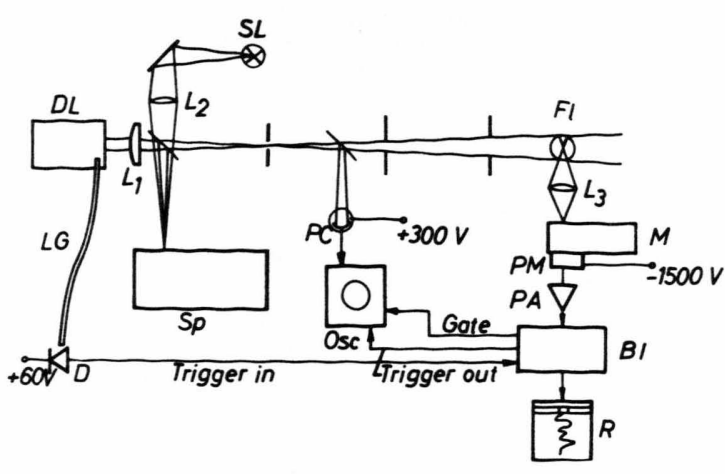

Fig. 3. Schematic diagram of the experimental setup used f'r laser excited atomic fluorescence flame spectrometry.

$\begin{array}{ll}\text { DL } & \begin{array}{l}\text { flashlamp-pumped tunable dye laser } \\ \text { (Zeiss, Oberkochen, Model 807101) }\end{array} \\ \text { SL } & \text { sodium vapor discharge lamp } \\ \text { Sp } & \text { spectrograph for coarse tuning of the dye laser } \\ \text { PC } & \text { photocell } \\ \text { Osc } & \text { dual trace oscilloscope } \\ \text { F1 } & \text { flame } \\ \text { M } & \text { grating monochromator } \\ \text { PM } & \text { photomultiplier tube } \\ \text { PA } & \text { Model 115 wideband preamplifier, PAR } \\ \text { BI } & \text { Model 160 boxcar integrator, PAR } \\ \text { R } & \text { chart recorder } \\ \text { LG } & \text { light guide } \\ \text { D } & \text { photodiode } \\ \text { L } \cdots L_{3} \text { lenses }\end{array}$

Coarse tuning of the rhodamine $6 \mathrm{G}$ laser to the $\mathrm{Na}-\mathrm{D}_{2}$-line was controlled with the aid of a metal discharge lamp and a 3 prism spectrograph. Fine adjustment was made by monitoring the fluorescence signal.

A conventional nebulizer-burner system (system of the ZEISS Flame Emission Photometer PF 5) was used for aspiration of the Na-containing solutions into the laminar-preximed flame and evaporation of the analyte. Though a $\mathrm{H}_{2}$-Argon-entrained air flame should be superior to a propane-air flame with respect to fluorescence reduction due to diminished quenching collisions,

Table 1. Characteristic data of the dye laser.

$T_{0}=$ peak transmission, $\mathrm{FWHM}=$ spectral halfwidth, $\mathrm{IF}=$ interference filter, $\mathrm{FP}=$ Fabry-Perot etalon.

\begin{tabular}{ll}
\hline laser resonator & $300 \mathrm{~mm}$ long, wedge shaped quartz plates with dielectric mirrors of $99.8 \%$ and $60 \%$ reflectivity \\
dye solution & $2 \times 10^{-4} \mathrm{M} / 1$ Rhodamine $6 \mathrm{G}$ in Methanol \\
pump energy & $10-13 \mathrm{Ws}$ \\
wavelength selector & IF (FWHM $=3 \mathrm{~nm}, T_{0} \approx 95 \%$ at $601 \mathrm{~nm}$ ) \\
& FP (solid quartz plate etalon, $0.25 \mathrm{~mm}$ thick, reflectivity $80 \%)$ \\
peak power & $20 \mathrm{~kW}$ for the laser narrowed with an IF alone \\
& $12.5 \mathrm{~kW}$ for the laser narrowed with an IF + FP \\
pulse length & $0.5 \mu \mathrm{sec}$ \\
spectral bandwidth & $0.2 \mathrm{~nm}$ for the laser narrowed by the IF alone \\
& $0.0033 \mathrm{~nm}$ for the laser narrowed by the IF + FP \\
\hline
\end{tabular}

* $\tau^{\text {sp }}=$ lifetime of excited level 2 for spontaneous emission. 
the propane-air flame was chosen. The former showed more signal fluctuations caused primarily by an increased evaporation of dust particles from the ambient air.

The laser-excited fluorescence radiation perpendicular to the direction of the laser beam was detected by a fast response photomultiplier (RCA 8645). Spectral isolation of the fluorescence signal against the flame background was achieved by a grating monochromator ( $f / 10$ aperture, dispersion $50 \AA / \mathrm{mm})$. Unless otherwise stated, a slit width of $0.1 \mathrm{~mm}$ was chosen for the detection channel, which is appropriate for the separation of the two $\mathrm{Na}-\mathrm{D}$ lines.

The pulse repetition rate of our dye laser could be continuously varied between $0.4 \mathrm{sec}^{-1}$ and $20 \mathrm{sec}^{-1}$. Most of the experiments were done with 1 to 3 laser pulses per second, which proved to be a good compromise between lifetime of the flashlamp and duty cycle of the source.

By use of a boxcar integrator for the amplification and integration of the photomultiplier output signal, noise originating from dark current, flame background fluctuations, and analyte emission is essentially reduced due to an appropriate choice of the off-to-on time of the gated amplifier with respect to the duty cycle of the laser. The remaining noise is mainly due to Mie and Rayleigh scattering of laser photons within the flame gases. Stable and proper synchronism between the fluorescence pulse and the aperture gate width of the integrator was possible if the light pulse of the flashlamp was used to trigger the gate. The integrated value of the fluorescence pulse was recorded on a strip chart recorder for evalution.

\section{Results and Discussion}

\section{a) Saturation irradiance}

To check the validity of the simple rate equation model for the estimation of the saturation irradiance, laser excited fluorescence and transmission were measured for a fixed concentration of the aspirated analyte while the exciting irradiance was lowered stepwise by neutral density filters over at least 3 orders of magnitude. Typical results for three different $\mathrm{Na}$ concentrations and the spectrally narrowed dye laser emission line (halfwidth $0.05 \AA$ ) are shown in Figure 4. The maximum laser output power corresponded to an irradiance of $25 \mathrm{~kW} / \mathrm{cm}^{2}$ for the fluorescence measurements and $17 \mathrm{~kW} / \mathrm{cm}^{2}$ for the transmission measurements.

Calculations of the saturation irradiance for the $\mathrm{Na}$ transition ${ }^{2} \mathrm{~S}_{1 / 2}-{ }^{2} \mathrm{P}_{3 / 2}$ have to consider the population of the $2 \mathrm{P}_{1 / 2}$ level built up by collisions of excited atoms in the $2 \mathrm{P}_{3 / 2}$ state with foreign gas molecules. The relative populations of the $2 \mathrm{P}_{3 / 2}$ state $\left(\mathrm{N}_{2}\right)$ and the ${ }^{2} \mathrm{P}_{1 / 2}$ state $\left(\mathrm{N}_{3}\right)$ can be determined experimentally by measuring the relative fluorescence intensities of the two $\mathrm{Na}-\mathrm{D}$ lines which could be excited by op- tical pumping of the ${ }^{2} \mathrm{~S}_{1 / 2}-2 \mathrm{P}_{3 / 2}$ transition with the dye laser. For our experimental conditions a ratio of $\mathrm{N}_{2}\left({ }^{2} \mathrm{P}_{3 / 2}\right) / \mathrm{N}_{3}\left(2 \mathrm{P}_{1 / 2}\right)=3$ was found. Within the flame, the lifetimes of the excited states are strongly shortened due to collisonal quenching. It follows from the cross section for quenching collisions $\pi \sigma_{\mathrm{q}}^{2}=25.4 \times 10^{-16} \mathrm{~cm}^{2}$ given by WINEFORDNER et al. 11 and a flame temperature of $2200^{\circ} \mathrm{K}$ that the collisional transition rate is $1.2 \times 10^{9} / \mathrm{sec}^{12}$. This value is about twenty times the transition rate for spontaneous emission. A value $\sigma_{12}=8.4 \times 10^{-13} \mathrm{~cm}^{2}$ for the absorption cross section of $2 \mathrm{~S}_{1 / 2}-2 \mathrm{P}_{3 / 2}$ transitions follows from 13 if the different statistical weights of the two $2 \mathrm{P}$ levels are considered. As the laser beam is linearly polarized due to the Brewster windows of the dye cell, $\alpha$ has to be set to 0.5 following from statistical orientations of the $\mathrm{Na}$ atoms with respect to the direction of polarisation. Using these parameters, a saturation irradiance

is calculated.

$$
I_{\mathrm{c}} \approx 0.4 \mathrm{~kW} / \mathrm{cm}^{2}
$$

Figure $4 \mathrm{a}$ demonstrates that the fluorescence signal at an irradiance level of $0.4 \mathrm{~kW} / \mathrm{cm}^{2}$ lies definitely

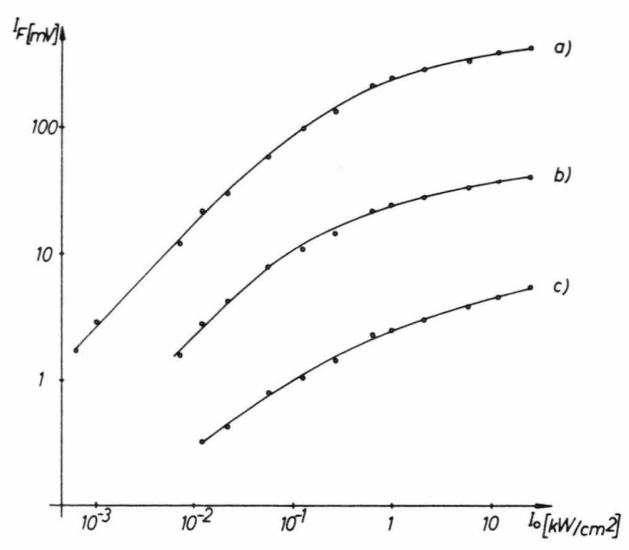

Fig. 4 a. Fluorescence signals observed in the $\mathrm{Na}-\mathrm{D}_{2}$ line as a function of the exciting laser radiation intensity for various Na-concentrations:

a) $10 \mu \mathrm{g} / \mathrm{ml}$, b) $1 \mu \mathrm{g} / \mathrm{ml}$, c) $0.1 \mu \mathrm{g} / \mathrm{ml}$.

below the value expected without saturation. From all 3 experimental curves for the different concentrations, it may be derived that about $30-35 \%$ of the saturation population is achieved, whereas the simple theoretical model predicts $50 \%$ of the limiting value. This agreement is satisfying taking into consideration the uncertainties of the parameters and remembering that the simple model neglects all line profile effects and the influence of nonquenching collisions. In spite of these simplifications, the model seems to be suitable for the estimation of the saturation irradiance. 
During the course of our experiments PIEPMEIER ${ }^{14,15}$ has published a more detailed theoretical analysis of the saturation problem in flame fluorescence spectrometry. He defined the ratio of the relative change in the excited atom fraction to the relative change in excitation irradiation as the regulation $G$. From our experimental result, a regulation $G=0.2$ is obtained for an irradiance level between 10 to $20 \mathrm{~kW} / \mathrm{cm}^{2}$. Assuming that all collisions between excited $\mathrm{Na}$-atoms and flame gas atoms result in collisional quenching and that no transitions between the $2 \mathrm{P}_{3 / 2}$ and $2 \mathrm{P}_{1 / 2}$ state are possible, Piepmeier has computed a value of $140 \mathrm{~kW} / \mathrm{cm}^{2}$ for Na. Both assumptions are not fullfilled for our experimental conditions. According to KILDAL and BYER ${ }^{13}$, the probability of quenching per collision at atmospheric pressure in air is 0.14 . Since $\mathrm{N}_{2}$ is also a major quencher in flames (besides $\mathrm{CO}$ and $\mathrm{CO}_{2}$ ), adoption of this number for an assessment of the necessary laser intensity to reach a regulation of 0.2 seems appropriate. Following the argument by PIEPMEIER, the quantity $F$ defined in 15 is approximately the fraction of all non-quenching collisions, i.e. $F=0.86$. With this value for $F$ only about $1 / 10$ of the laser energy energy density is needed to reach the same regulation of 0.2 as when nearly all collisions quench (for comparison see Fig. 3 of 15 ). This result coincides favourably with our observations, though we cannot claim quantitative agreement. The values chosen in 15 for the different parameters and in the calculations, do not necessarily coincide with our experimental parameters.

\section{b) Analytical curves and detection limits}

Two different analytical curves (log-log-plots), which were obtained by excitation of the Na-atoms with laser emission lines of different spectral bandwidth $\left(A: \Delta \lambda_{\mathrm{L}}\right.$ $=0.033 \AA, \mathrm{B}: \Delta \lambda_{\mathrm{L}}=2.0 \AA$ ) but the same total intensity are shown in Figure 5. Both curves are linear from the detection limits up to concentrations, which are larger by at least $5 \times 10^{4}$. Whereas the spectral power densities of the two excitation lines differ by a factor of about 60 , the observed fluorescence signal differs only by a factor of 2.2. Taking into account the absorption bandwidth of $0.1 \AA$, the fluorescence intensity excited by the narrower laser line should be at least 15 times greater**. The apparent discrepancy can be explained by the saturation effects. From the experimental curves (Fig. $4 \mathrm{a}$ ) a reduction of the fluorescence radiation by a factor of 7 may be derived for the employed irradiance of $5 \mathrm{~kW} / \mathrm{cm}^{2}$.

In spite of the small increase in fluorescence signal, excitation by the narrow band laser is to be preferred if frequency fluctuations of the laser line are small

** (Assuming that the absorption profile is described by a Lorentzian function).

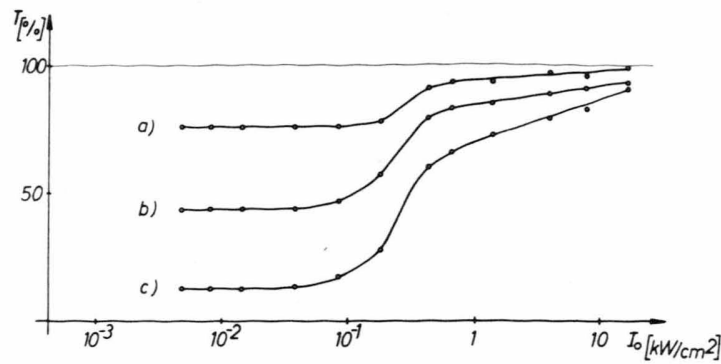

Fig. 4b. Transmission factor $T$ of the illuminated flame as a function of the incident irradiation $I_{0}\left(\mathrm{~kW} / \mathrm{cm}^{2}\right)$. Parameter: Na-concentration:

a) $10 \mu \mathrm{g} / \mathrm{ml}$, b) $50 \mu \mathrm{g} / \mathrm{ml}$, c) $100 \mu \mathrm{g} / \mathrm{ml}$.

compared with the absorption bandwidth for the following reasons:

1. As the sensitivity of the method is severely limited by scatter of laser photons by aerosols and molecules, and refractive index variations within the flame gases, the ratio of the fluorescence to the scattered radiation intensity decreases with increasing bandwidth of the exciting radiation.

2. Influence of laser power fluctuations on the signal can be reduced if slightly saturated conditions can be produced within the vapor phase.

3. The deviations from the linear relation between concentration and fluorescence signal, which is mainly due to reabsorption of the fluorescence radiation, is less marked for the curve obtained with the narrow

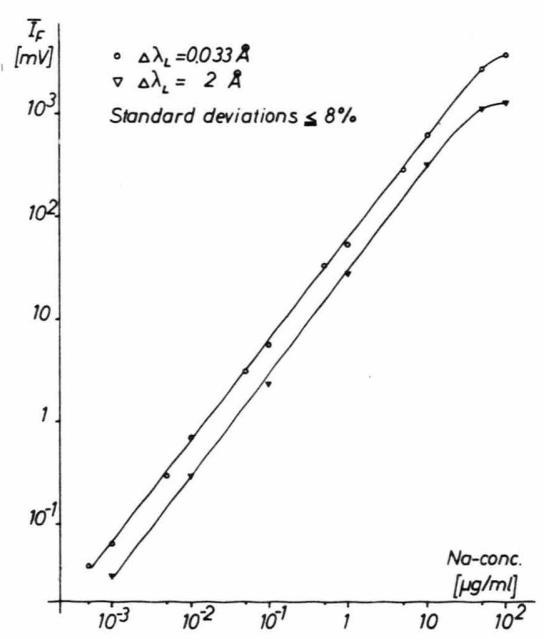

Fig. 5. Analytical curves for laser excited atomic fluorescence of $\mathrm{Na}$ in a propane/air flame. The two curves were obtained by using laser emission lines of different bandwidths $\Delta \lambda_{\mathrm{L}}$ for excitation. 
laser line. This effect can be explained by the fact that irradiation with intensities well beyond the saturation irradiance causes strong bleaching of the atomic ground state.

Recordings showing typical signal-to-noise ratios for Na-concentrations near the limit of detection are shown in Figure 6. These measurements were taken at

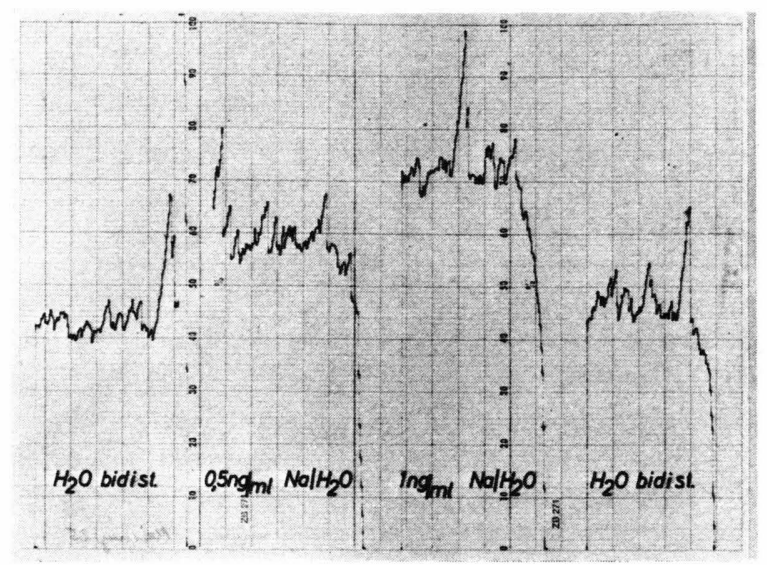

Fig. 6. Typical recordings of the fluorescence signal and the background (scattered) radiation near the limit of detection.

$100 \%$ corresponds to $5 \mathrm{mV}$.

5-6 $\mathrm{mm}$ above burner top. The lowest detectable concentration resulting in a signal-to-noise ratio of 2 is about $0.2 \mathrm{ng} / \mathrm{ml}$. This detection limit is comparable to the best reported value of $0.1 \mathrm{ng} / \mathrm{ml}$ in $\mathrm{AE}$ flame spectrometry 16 . The high spikes of the noise are caused by flashes of evaporated dust particles and were disregarded for the evaluation of the detection limit.

It is interesting that the blank signal was partly produced by traces of sodium in the solvent (bidistilled water). This has been verified by comparing the blank signals measured at $589.0 \mathrm{~nm}\left(\mathrm{Na}-\mathrm{D}_{2}\right)$ and at a slightly different wavelength $(\sim 580 \mathrm{~nm})$. At $580 \mathrm{~nm}$, no difference in the scattered radiation was observed whether water or nothing was being aspirated.

\section{c) Fluorescence line profile}

By scanning the dye laser emission line stepwise across the $\mathrm{Na}-\mathrm{D}_{2}$ line, the fluorescence line profile shown in Fig. 7 was recorded.

The wavelength scale was calculated from the angular position of the tilted Fabry-Perot interferometer. The wavelength $\lambda_{0}$ for the maximum of the fluorescence signal was assumed to be $5889.96 \AA$ neglecting the small Lorentz shift of the line center of $0.02 \AA 17$.

The halfwidth of the recorded line profile corresponded to a wavelength interval of $0.12 \AA$. Taking

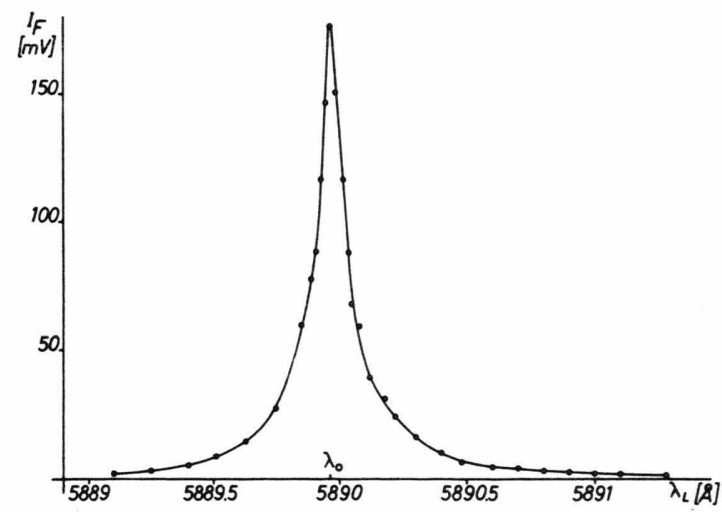

Fig. 7. Fluorescence line profile of the $\mathrm{Na}-\mathrm{D}_{2}$ line scanned by a $0.033 \AA$ wide exciting laser line. $\mathrm{Na}$-concentration: $10 \mu \mathrm{g} / \mathrm{ml}$.

into consideration the finite linewidth of the scanning laser, a corrected value for the halfwidth of $0.09 \AA$ may be derived.

From the hyperfine splitting of the $\mathrm{Na}-\mathrm{D}_{2}$ line, the Doppler width corresponding to the flame temperature of $2200^{\circ} \mathrm{K}$ and the broadening of the Doppler line by collisions within the flame, a halfwidth of $0.08 \AA$ would be expected.

\section{Conclusions}

This investigation of dye laser excited flame fluorescence spectrometry has shown that saturated conditions within a $\mathrm{Na}$ vapor sample occur at modest levels of laser output power densities. A simple theoretical model based on rate equations enables the experimentator to estimate the useful irradiance level. A saturation irradiance $I_{c}$ may be defined for which the population of the excited level reaches $50 \%$ of the limiting value. $I_{\mathrm{c}}$ decreases with increasing absorption cross section for the transition from the ground state to the excited level and increases with decreasing relaxation times within the atom.

With flashlamp-pumped dye laser sources which are commercially available, output intensities which should be sufficient to get saturated conditions in the resonance lines for many elements could be obtained over the entire visible part of the spectrum from $430 \mathrm{~nm}$ to $700 \mathrm{~nm}$. By frequency doubling of the visible dye laser emission with ADP and KDP crystals 18 , it is possible to generate power densities of the order of some $\mathrm{kW} / \mathrm{cm}^{2}$ even in the wavelength range from $261 \mathrm{~nm}$ to $350 \mathrm{~nm}$.

Initial experiments with these frequency doubled sources for the analytical detection of $\mathrm{Pb}, \mathrm{Mg}$ and $\mathrm{Ni}^{19}$ have indicated that saturation effects become observable even for resonance lines which have lower f-values than the $\mathrm{Na}-\mathrm{D}$ lines. 
Long linear analytical curves (over 5 orders of magnitude of analyte concentrations) have been obtained for $\mathrm{Na}$. The detection limit of $0.2 \mathrm{ng} / \mathrm{ml}$ is comparable to the best previously reported values in the literature. Further improvement of the sensitivity in laser excited fluorescence spectrometry seems possible if fluctuations of the dye laser output can be reduced or if such instabilities can be cancelled by recording the ratio of the fluorescence signal and the laser output power.*

\section{Acknowledgement}

The work on which this report is based was supported by the Bundesminister für Bildung und Wissenschaft as part of the technology program of his ministry. The authors are exclusively responsible for the contents of this report.

\section{* Note added in proof:}

After completion of our manuscript, J. D. Winefordner informed us that he and his co-workers have studied the nonlinear effects in AFS by employing a $\mathrm{N}_{2}$-laser pumped dye laser (bandwidth $0.1-1 \mathrm{~nm}$ ) as excitation source. The results of their investigations will be published in two papers submitted to Spectrochimica Acta B: (N. Omenetto, P. Benetti, L. P. Hart, J. D. Winefordner, C. Th. Alkemade, "Nonlinear Optical Behavior in Atomic Fluorescenc Flame Spectrometry", and N. Omenetto, L. P. Hart, P. Benetti, J. D. Winefordner, "On the Shape of Atomic Fluorescence Analytical Curves with a Laser Excitation Source" Spectrochim. Acta 28 B, 289 [1973] and 28 B, 301 [1973]).

\section{References}

1 J.D. Winefordner and R.C. ELSER, Analyt. Chem. 43, 24a (1971).

2 J.D. WingFordner and T.J. ViCKers, Analyt. Chem. 42, 206 R (1972).

3 L.M. Fraser and J.D. Winefordner, Analyt. Chem. 43, 1693 (1971) and Analyt. Chem. 44, 1444 [1972].

4 M. B. Denton and H.V. Malmstadt, Appl. Phys. Letters 18, 485 (1971).

5 J. Kuhl and G. Marowsky, Opt. Comm. 4, 125 (1971).

6 M. Hercher, Appl. Opt. 6, 947 (1967).

7 F. GIREs, IEEE J. of Quantum Elec. 2, 624 (1966).

8 J. KUHL, G. MarowsKY, P. KUNSTMANN, and W. SCHMIDT, Naturforsch. 27 a, 601 (1972).

9 W. SCHмidT and N. WITTEKINDT, Zeiss Informationen 80, 33 (1972).

10 W. Schmidt, Physik in unserer Zeit 3, 164 (1972).

11 R.F. BROWNER and J.D. WINEFORDNER, Spectrochim. Acta 27B, 257 (1972).

12 A.C.G. Mrtchell and M.W. ZeEmansky, Resonance Radiation and Excited Atoms, Cambridge 1961.

13 H. Kildal and R. L. Byer, Proc. IEEE 59, 1644 (1971).

14 E. H. PiePMeIER, Spectrochim. Acta 27 B, 431 (1972).

15 E. H. PiePMEIER, Spectrochim. Acta 27 B, 445 (1972).

16 J.D. Winefordner, V. Svoboda, and L.J. Cline, CRC Crit. Rev. Anal. Chem. 1, 233 (1970).

17 J. B. Willis, Spectrochim. Acta 26B, see e.g. 177 (1971).

18 J. Kuhl and H. Spitschan, Opt. Comm. 5, 382 (1972) and the literature cited there.

19 J. KuHL and H. Spitschan, Opt. Comm. 7, 256 (1973). 